\title{
Buscando arquitecturas para una ciudad saludable. El caso del sanatorio escalonado en los años veinte
}

\author{
Seeking architectures for a healthy city. The \\ stepped sanatorium case study in the twenties
}

Resumen

Autores:

Eduardo Jiménez-Morales* eduardo_jm@uma.es

Ingrid Carolina Vargas-Díaz** carovar@correo.ugr.es

*Universidad de Málaga ** Universidad de Granada

Recibido: 14/Mar/2021 Aceptado: 07/Jul/2021
A I inicio del siglo XX algunos médicos y arquitectos centraron sus esfuerzos en buscar soluciones a los graves problemas de hacinamiento e insalubridad que sufrían los habitantes de la ciudad industrial. Para ello trataron de promover un hábitat higiénico basado en un cambio de las formas arquitectónicas que definían tanto el espacio doméstico como el urbano. La arquitectura de sección escalonada fue entonces utilizada de manera recurrente como modelo preliminar para alcanzar estos objetivos. No obstante, a pesar de su importancia, son escasos los estudios que han profundizado en las circunstancias que hicieron de ella todo un referente arquitectónico con el que imaginar la ciudad saludable en los años 20 , o en el papel protagonista que desempeñaron ciertas arquitecturas hospitalarias a la hora de promover ese cambio. El sanatorio antituberculoso de terrazas retranqueadas es un caso ejemplar en este sentido y, por ello, es objeto de estudio en este artículo.

Palabras clave: ciudad saludable; vivienda social; sanatorio; arquitectura escalonada; Europa entreguerras

\section{Abstract:}

At the beginning of the 20th century some doctors and architects focussed their efforts on finding solutions to improve the serious problems of overcrowding and unhealthy living conditions in the industrial cities. For this purpose, they promoted a healthier urban environment and suggested different changes in the architectural design that could define the inner and urban space. To achieve this, one of the most widespread models in the 1920s was the stepped-back architecture. However, despite its importance, few studies have examined the circumstances that made this model an architectural reference for a healthier city and the role-played by certain hospital architecture in promoting those changes. Therefore, the aim of this article is to analyse one of the most outstanding architectural models in this transition: the stepped sanatorium.

Keywords: healthy city; social dwelling; sanatorium; stepped architecture; interwar Europe 


\section{Introducción}

Mucho antes de que el Covid-19 pusiera en tela de juicio la idoneidad de los paradigmas arquitectónicos y urbanísticos contemporáneos para contener la propagación de la pandemia (Megahed y Ghoneim, 2020), otra enfermedad, la tuberculosis, ya había hecho lo propio a finales del siglo XIX y principios del XX. El hacinamiento y la insalubridad que azotaban a la población de la ciudad industrial dibujó entonces el escenario idóneo para la incubación y la propagación de esta letal enfermedad, así como para la prolífica producción de modelos urbanos reformistas que promulgaban un regreso a la vida en el campo y el reencuentro con la naturaleza como fuente de bienestar físico y espiritual. El modelo de la ciudad jardín propuesto por Howard (1898) en su obra To-morrow: a Peaceful Path to Real Reform, culminó esta corriente de pensamiento crítico contra la ciudad industrial que fue tomando forma en Gran Bretaña durante el siglo XIX, aunque en sí mismo no aportó ninguna solución para que este ansiado reencuentro con la naturaleza también fuera posible en el corazón de la ciudad densa y contaminada.

Otras utopías sociales, en cambio, si estuvieron atentas a estas deficiencias. Godin (1871), por ejemplo, procuró que los individuos estuvieran expuestos a los beneficios saludables del sol en el espacio doméstico del Famililistère. Borie (1865), por su parte, incorporó la naturaleza al espacio urbano a partir del estudio del perfil transversal de las calles, o de la orientación y la forma de los edificios, en un intento por sanear los barrios periféricos de París. En esa misma dirección trabajaron autores como Knauff (1879), Vogt (1879) o August Vorrher, quien recurrió a los principios teóricos del Sonnenbaulehre (Faust, 1829) con objeto de alcanzar una máxima exposición solar del tejido urbano a partir de un adecuado trazado viario o una estudiada disposición de los edificios en su plan para la expansión urbana de Múnich en 1821.

La popularización de estos criterios de diseño para el espacio doméstico y el urbano estuvo acompañada por los avances médicos $y$ fisiológicos del final de siglo. El descubrimiento del origen bacteriológico de la tuberculosis, que llegó a exterminar a alrededor de un cuarto de la población europea (Duarte, 2005), refrendó el papel que jugaba la adecuada ventilación y el asoleo del hábitat para su prevención, y convirtió al eslogan Licht und Luft (aire y luz) en el ideal higiénico para el diseño arquitectónico y urbanístico al inicio del siglo XX (Campbell, 2005). En este contexto reformista, la arquitectura del sanatorio, como institución especializada en la lucha contra la enfermedad (Cremnitzer y Toulier, 2008; Grandvoinnet, 2020; JiménezMorales, 2014; Jiménez-Morales y Vargas-Díaz,
2017), acabó convertida en el prototipo en torno al cual giró el debate tipológico (Campbell, 2005; Cremnitzer, 2005; Iglesias Picazo, 2011; Overy, 2008) y metodológico (Colomina, 2019) en el seno del Movimiento Moderno.

No obstante, dentro de este conjunto de arquitecturas hospitalarias dedicadas a la cura de la tuberculosis, fue el sanatorio escalonado el que suscitó mayor interés en un comienzo, ya que su perfil escalonado permitía una mejora de las condiciones de habitabilidad al proporcionar más soleamiento y aire puro, tanto a la vivienda colectiva como al espacio urbano. Así, sus características terrazas retranqueadas se usaron sistemáticamente con el fin de otorgar una respuesta higienista a las inquietudes que suscitaban los problemas de salud pública en el seno de la ciudad industrial. Los proyectos parisinos de Henri Sauvage para los immeubles à gradins (1912-1928), las viviendas para la Inzerdorferstrasse (1923) de Adolf Loos en el marco del Plan Regulador de Viena, los dibujos de Garnier (1917) para la Cité Industrielle (1904-1917) o el utópico Wohnberg Project (1928) de Walter Gropius, entre otros diseños destacados, han dado buena muestra de ello.

Sin embargo, a pesar de su importancia, son escasos los estudios que, a día de hoy, han profundizado en las condiciones que dieron forma al sanatorio escalonado al inicio del siglo XX, o en las circunstancias que hicieron de él un primer referente arquitectónico con el que imaginar la ciudad saludable en los años veinte. Para subsanar este déficit, este artículo propone abordar primero la aparición de este prototipo como resultado de la adaptación de la arquitectura original del sanatorio a las necesidades médicas, condicionadas por la efectividad de las terapias. A continuación, estudia el contexto urbano en el que se promovió un hábitat higiénico y una experimentación tipológica con base en la arquitectura sanatorial tras la Primera Guerra Mundial. Para finalizar, se exponen las principales conclusiones así como las futuras líneas de investigación.

\section{Metodología}

El objeto de estudio de la investigación es, por tanto, la génesis y desarrollo temprano del sanatorio escalonado hasta el momento en que sus criterios de diseño se dieron por sentados e inspiraron a las corrientes reformistas que promovían un hábitat higiénico y saludable. El método de investigación es, en consecuencia, analíticosintético, deductivo-inductivo y cronológico; que además es convergente con el estudio historiográfico de la arquitectura para un período cronológico específico. El método igualmente es experimental y fundamentalmente genealógico. Una fórmula de análisis un tanto compleja que atiende a los cambios que experimenta la arquitectura en su dimensión material, funcional y estilística, así como en su propia conceptualización, en concordancia con los distintos contextos y períodos históricos. En este caso su aplicación ha sido además múltiple, pues solo a partir de la imbricación de distintos procesos de transformación es posible recomponer la genealogía arquitectónica del objeto de estudio.

La escasa documentación científica de referencia y la dificultad para realizar un estudio comparativo tan amplio, 
obligó al manejo de fuentes primarias y secundarias, bibliográficas y archivísticas, no siempre relacionadas con la arquitectura. El estudio documental sobre el sanatorio, por ejemplo, incluye publicaciones afines a campos disciplinares tan dispares como la medicina, la sociología o la historia. En lo que atañe exclusivamente a su arquitectura, se consultó a Cremnitzer (2005), Cremnitzer y Toulier (2008), Grandvoinnet (2020), Jiménez-Morales y Vargas-Díaz (2017), junto a autores como Miller (1992), que constituyen referencias básicas sobre esta tipología hospitalaria. Para reconocer las particularidades del contexto suizo, se acudió a JiménezMorales (2014) o Lüthi (2005), quienes centran una parte de sus trayectorias investigadoras en torno a los procesos de formalización arquitectónica del sanatorio alpino.

A la hora de evaluar los vínculos que existen entre la arquitectura sanatorial y el resto de tipologías durante el período entreguerras se estudian manuales y libros recopilatorios de la época. Caben destacar las referencias de Döcker (1929), Gescheit (1929), Giedion (1929) o Grober (1932) que se completan con fuentes archivísticas por autor o caso de estudio, monografías o revistas especializadas sobre arquitectura. Asimismo, se consulta la literatura reciente afín al tema de estudio, con especial atención a Campbell (2005), Colomina (2019), Iglesias Picazo (2011) y Overy (2008), quienes revelan los progresos de la arquitectura de vanguardia desde la obsesión médica dominante en los inicios del siglo XX. Su influjo sobre el ideal habitable en la ciudad europea de la época marcó un horizonte para recuperar su condición salubre, tal como describen Dumont (1991), Medici (2003) o Sonne (2009) entre otras fuentes bibliográficas básicas.

\section{Resultados}

\subsection{El Freilufthaus y la terapia solar}

Con la convicción decimonónica de que la ciudad industrial era el caldo de cultivo ideal para muchas enfermedades debido a sus insalubres condiciones de habitabilidad, también surgió la idea de que existían, como contrapunto, entornos naturales saludables alejados del contexto urbano donde recuperar la salud quebrada. El viaje estacional a la montaña en general, y a los Alpes suizos en particular, fue un claro ejemplo de ello, convirtiéndose en destinos sanitarios de éxito internacional en muy poco tiempo. La popularización de las terapias médicas antituberculosos con base en la cura al aire libre que allí se practicaban fueron las responsables de este fenómeno migratorio en su inicio, así como de la consolidación tipológica del sanatorio como resultado de desarrollo de destacadas innovaciones arquitectónicas bajo el dictado de las necesidades médicas (JiménezMorales y Vargas-Díaz, 2017).

La Liegehalle o terraza de cura colectiva, fue una de estas aportaciones. $Y$ aunque originalmente se concibiera en 1876 para facilitarle a los enfermos el acceso al aire libre desde su propia habitación, no tardó en transformarse en una parte indispensable del sanatorio y de una gran variedad de tipologías arquitectónicas hasta nuestros días (Overy, 2008). No obstante, a comienzos de siglo XX, la evolución de las terapias antituberculosas produjo un nuevo cambio en la formalización arquitectónica de sanatorio que centra el interés de esta investigación. Concretamente desde 1902, cuando los tratamientos que desarrollan los doctores Oskar Bernhard y Auguste Rollier comienzan a combinar la cura de aire con el efecto saludable de la exposición directa del paciente a la radiación solar.

La necesidad de una amplia terraza que facilitase el baño solar, sin sombras arrojadas y orientada al sur, impulsó esta evolución en su arquitectura. Así, las primeras clínicas destinadas a la helioterapia, muchas de ellas situadas en la localidad suiza de Leysin, incorporaron varios niveles de terrazas de cura encastradas en su fachada sur pero sin la amplitud suficiente para disponer las camas de los paciente al sol. Unas deficiencias en parte subsanadas con la aparición de las terrazas solárium que complementaban la función que ya desempeñaba la tradicional Liegehalle, tal y como sucedió en la clínica Les Chamois (1909) del doctor Rollier. Su atípica ubicación en una fachada sur-oeste respondió extraordinariamente bien a la necesidad de ejecutar el baño solar a bajas temperaturas (Rollier, 1923), condición elemental para llevar a cabo la terapia de este reconocido médico suizo.

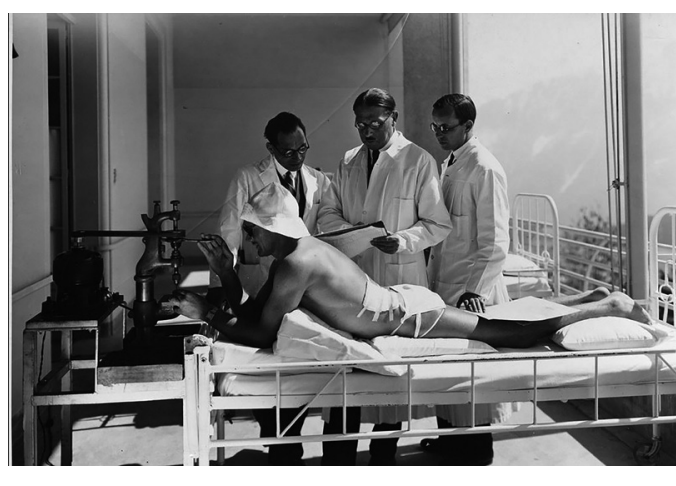

Figura 1: El Dr. Auguste Rollier (centro) examina a un paciente sometido a helioterapia en la Clínica Miremont, Leysin, 1911

Fuente: Fondation Miremont, (2017). Wikimedia. (CC BYSA 4.0)

No obstante, fue en la institución de helioterapia de Le Frênes (1911) donde Rollier llevó al límite esta aportación arquitectónica con una amplia azotea solárium meridional distribuida en dos niveles escalonados (Figura 1). Un diseño que seguramente estuvo inspirado en la planimetría que acompañaba a la ponencia que presentó el doctor David Sarason en el XIV Congreso Internacional de Higiene y Demografía de Berlín (1907) y con las que dio a conocer una innovadora tipología arquitectónica para hospitales y viviendas. Bajo el nombre de Freilufthaus, Sarason (1907) rompió buena parte de los dogmas establecidos al proponer un sanatorio en vertical con un singular escalonamiento de su fachada meridional (Figura 2). El Freilufthaus además podía ser extrapolable a cualquier otra edificación hospitalaria o residencial, generalizando así las condiciones para un hábitat saludable. 


\section{Freiluft $=$ Sanatorium}

für 72 Betten

6 Zimmer à 1 Bett

14 Zimmer à 2 Betten

2 Zimmer à 3 Betten

2 Zimmer à 4 Betten

3 Säle à 8 Betten
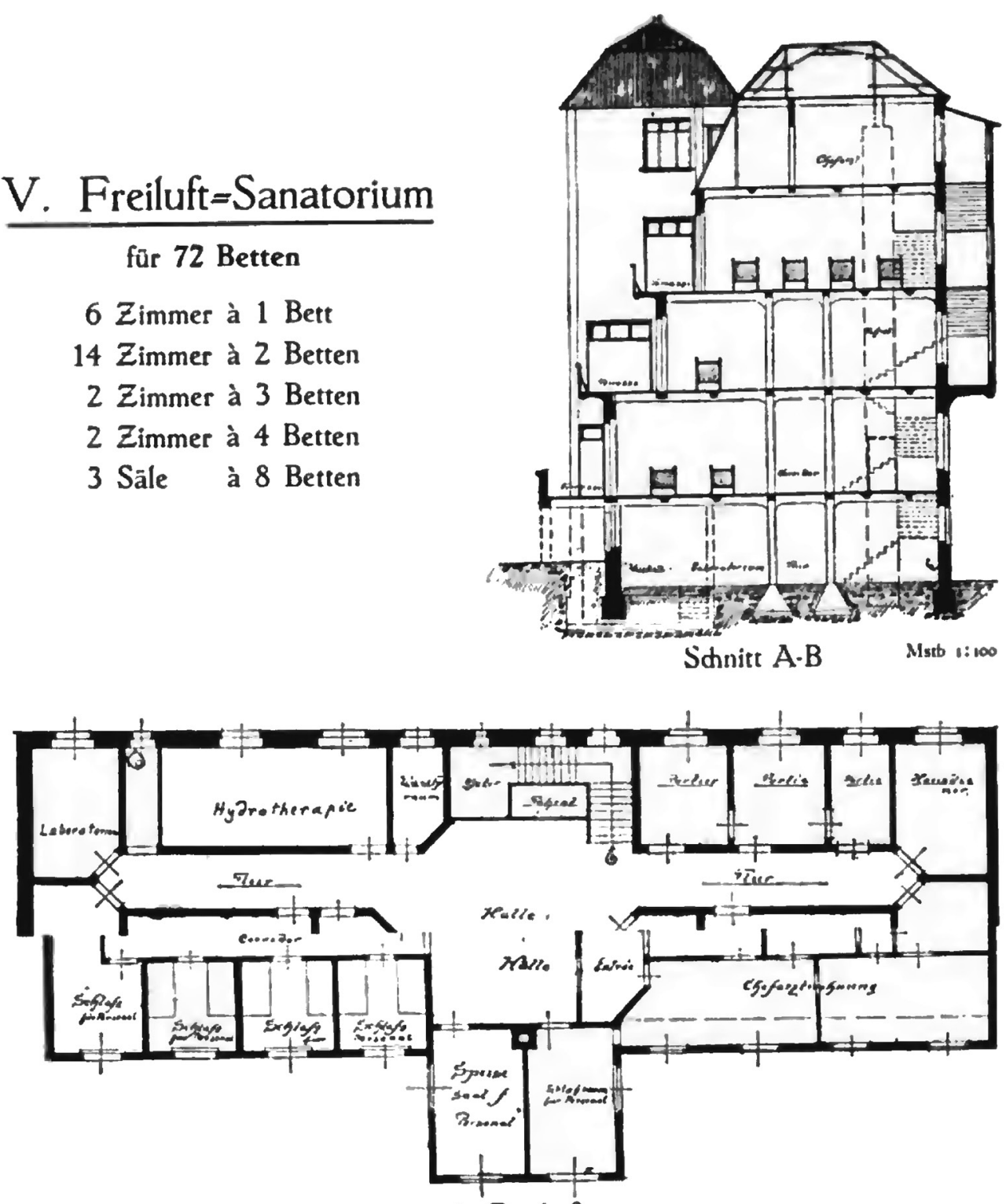

4. Geschoß

Figura 2: Planta y sección transversal del Freilufthaus, 1907

Fuente: D. Sarason, (1913). Internet Archive. Public Domain Mark 1.0

Evidentemente, el diseño escalonado en altura permitía disponer de terrazas soleadas accesibles desde cada habitación. Aunque para su materialización, era indispensable desarrollar técnicas constructivas que permitiesen romper el plano estructural de la fachada sin socavar toda la estabilidad del edificio. Una buena parte de estas técnicas estaban relacionadas con la incorporación del hormigón armado como material de construcción estructural, tal y como vaticinó Sarason (1909). No obstante, cuando en 1908 se consiguió edificar parcialmente el Freilufthaus en el Children Sanatorium de Lausana (Suiza), los ideales reformistas que representaba este tipo arquitectónico impulsaron una revisión del diseño original de otros muchos sanatorios. Fue el caso del Wald Sanatorium, con el solárium obra de Arthur Wiederanders y Walther Koch, o la azotea que sustituyó al bajo cubierta de la clínica suiza Clavadel en torno al año 1910.
Pero la propuesta más innovadora fue sin duda la ampliación de la clínica del doctor Bernhard en la localidad suiza de St. Moritz (1917) (Figura 3). Bajo la dirección del arquitecto alemán Nicolaus Hartman The Younger, se proyectó este Freilufthaus de cinco niveles, cuatro de ellos reservados para las habitaciones de los enfermos. Además, con el objetivo de compensar los perjuicios que ocasionaba la disminución de la profundidad de las estancias en cada nivel, se introdujo un corredor interior de distribución. Se conseguía así una proporcionalidad en las dimensiones de las estancias, en su asoleo y ventilación. Por último, se utilizó una gran cubierta solárium para coronar el proyecto, con un sistema interior de drenaje que compartía características con el que diseñó el constructor alemán Samuel Häusler en 1851 (Poeschel, 1928) y con el que esbozó en 1927 el arquitecto Le Corbusier en sus propios cuadernos de trabajo. 


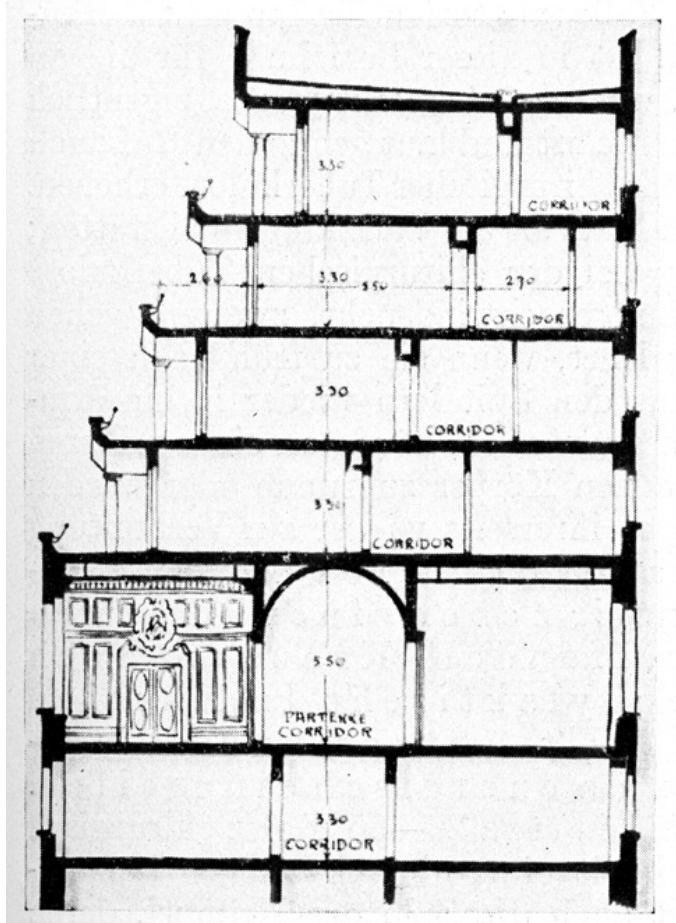

Fig. 63. Schnitt: Maßstab 1:100 (zu Fig. 61)

Figura 3. Sección transversal de Bernhard Klinic en St. Moritz, 1917

Fuente: O. Bernhard, (1917)

Lamentablemente, el estallido de la Primera Guerra Mundial condenó a este y muchos otros proyectos a ámbito de las publicaciones teóricas o el de las obras no realizadas. Hubo que esperar hasta el período que quedó definido entre las dos guerras mundiales para que la arquitectura de perfil escalonado se materializase en el ámbito sanitario porque, paradójicamente, antes de la Primera Guerra Mundial ya se había llevado a cabo la primera aplicación del Freilufthaus en una vivienda colectiva parisina de la mano de los arquitectos galos Henri Sauvage y Charles Sarazin (1912-1913). Para entonces, ciertos médicos y arquitectos alemanes estaban ya analizando críticamente buena parte de las innovaciones arquitectónicas que había aportado la arquitectura hospitalaria desde 1900, evaluando, a su vez, su transferencia a la vivienda colectiva como instrumento para higienizar la ciudad.

\subsection{La vivienda como un sanatorio dentro de la ciudad}

Con la celebración del Congreso Internacional de la Tuberculosis de Paris de 1905, al fin se consolidó tipológicamente el sanatorio como institución especializada en el tratamiento de la enfermedad. No obstante, también se escucharon las primeras voces críticas que apostaban por la prevención de la tuberculosis dentro de la ciudad antes que promover recintos hospitalarios lejos de los entornos urbanos. Una posición que estaba justificada por que este tipo de instalaciones resultaban onerosas para las arcas públicas y por el malestar psíquico que sufrían muchos de los pacientes, ya que permanecían aislados de sus entornos domésticos durante largos períodos de tiempo; a lo que había que añadir que para luchar contra la tuberculosis, considerada aún hoy una enfermedad social, era además imprescindible actuar decididamente sobre las condiciones insalubres del hábitat en la ciudad.

Así lo entendía el doctor Camile Savoire, quien ensalzó los planteamientos de su homólogo alemán David Sarason durante su disertación en la École des Beaux-Arts de París bajo la presidencia de Louis Bonnier, director de los Servicios de Arquitectura de la Villa de París y responsable último de su planificación urbana. El doctor Savoire destacó las excelentes condiciones arquitectónicas del Freilufthaus para garantizar el asoleo y la ventilación en un mínimo espacio construido, así como su versatilidad para utilizarse como modelo para alcanzar el ideal higiénico que ellos estaban buscando para la vivienda colectiva (Savoire, 1902); cualidades visibles tanto en el espacio doméstico como en el urbano, puesto que el progresivo retranqueo en altura del plano de fachada modificaba además la sección habitual de la calle.

No obstante, la posibilidad de convertir un tejido residencial decimonónico en un parque de viviendas colectivas y salubres de perfil escalonado estaba aún lejos de ser real. Solo bastaba con observar las planimetrías publicadas por el doctor Sarason para comprobar las limitaciones del Freilufthaus. Por un lado, la reducción progresiva de la superficie construida en cada nivel terminaba limitando la altura máxima del edificio que, por lo general, solo podría tener un total de cinco plantas. A la vez, las habitaciones situadas en los niveles inferiores eran profundas en exceso, afectando negativamente a una adecuada ventilación e iluminación. Así lo explicó Grober (1932) en su libro Das deutsche Krankenhaus, donde el médico alemán además valoraba críticamente otras muchas soluciones arquitectónicas hospitalarias aportadas por el sistema sanitario alemán desde 1900.

La alternativa al prototipo escalonado podía encontrarse en el urbanismo, flexibilizando las ordenanzas municipales, tal y como hizo Louis Bonnier a partir de 1913, o teorizando sobre las posibles relaciones geométricas entre la orientación, la separación y la altura de los edificios que asegurasen un adecuado soleamiento. En este sentido, el arquitecto Adolphe Augustin Rey concluyó en 1912 que la separación entre los edificios meridionales debía de ser casi el doble que la de los mismos edificios orientados hacia este u oeste; principios que formaron parte de su posterior teoría helio-térmica (Rey et al., 1928) y que tuvo, a su vez, gran influencia sobre otros arquitectos, como Le Corbusier, quien presentó en el III CIAM su plan para la Ville Radieuse tomando como base el método de orientación de Rey.

En cualquier caso, acompañando a la descripción de Freilufthaus, Grober (1932) se hizo eco del proyecto del arquitecto alemán Richard Döcker para el Waiblingen County Hospital hacia 1928 (Figura 4). Un edificio lineal de dos plantas, con un original doble juego de retranqueos y voladizos en su sección, que solucionaba la falta de luz y de ventilación que existía en las habitaciones de los niveles inferiores del modelo del doctor Sarason. Para ello, Döcker había tomado la idea del corredor interior de Nicolaus Hartman The Younger (Figura 3) para desplazarlo después al perímetro del edificio, conservando así la 
proporcionalidad en la dimensión de las habitaciones. El resultado fue una doble fachada escalonada, con dos niveles de terrazas, que tenía una marquesina angulada para regular la incidencia de los rayos del sol en el interior de la habitación a lo largo del año.

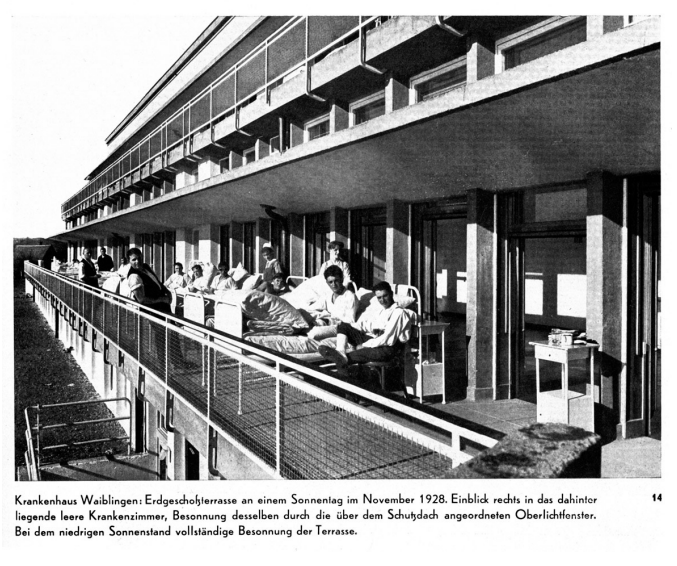

Figura 4: Pacientes en la terraza del County Hospital de Richard Döcker en Waiblingen, 1928

Fuente: R. Döcker, (1929)

El Waiblingen County Hospital aparece publicado en la sección que Döcker (1929) dedicó a la arquitectura hospitalaria en su libro Terranssen-typ. Junto a él, los proyectos del doctor Sarason o del arquitecto Tony Garnier, con su prototipo de Sanatorio Franco-Americano, aparecían reseñados dentro de un amplio catálogo de combinaciones arquitectónicas escalonadas con el que el autor pudo demostrar las posibilidades que ofrecía la arquitectura en terrazas para proporcionar un hábitat saludable en la ciudad, permitiendo su apertura al so y su reencuentro con la naturaleza y el paisaje (Döcker, 1929); ideales que compartía con autores como Gescheit (1929) o Giedion (1929), quienes igualmente publicaron la obra del arquitecto alemán junto a las del sanatorio suizo Queen Alexandra (1909) de Haefeli y Pfleghard, o Zonnestraal (1919-40), el emblemático sanatorio holandés de Duiker y Bijvoet.

Evidentemente, la prolífica difusión de esta arquitectura sanitaria no tardó en traducirse en novedosos prototipos residenciales que, en muchos casos, tenían sus precedentes formales en alguna de las obras de arquitectos como Henri Sauvage o Tony Garnier. Este último demostró desde el comienzo de su carrera su inquietud por concebir un espacio doméstico saludable. Así lo acredita su propuesta para el Concurso de la Fundación Rothschild de Paris en 1905 (Figura 5), con un edificio plurifamiliar de viviendas sociales cuyo trazado zigzagueante norte-sur redefinía el límite del espacio libre al que se asomaban todas las estancias. Garnier conseguía así democratizar el acceso al aire libre y al sol, rompiendo la ordenación urbana en manzana cerrada y patio interior, e independizar el diseño del inmueble del trazado original de las calles circundantes.
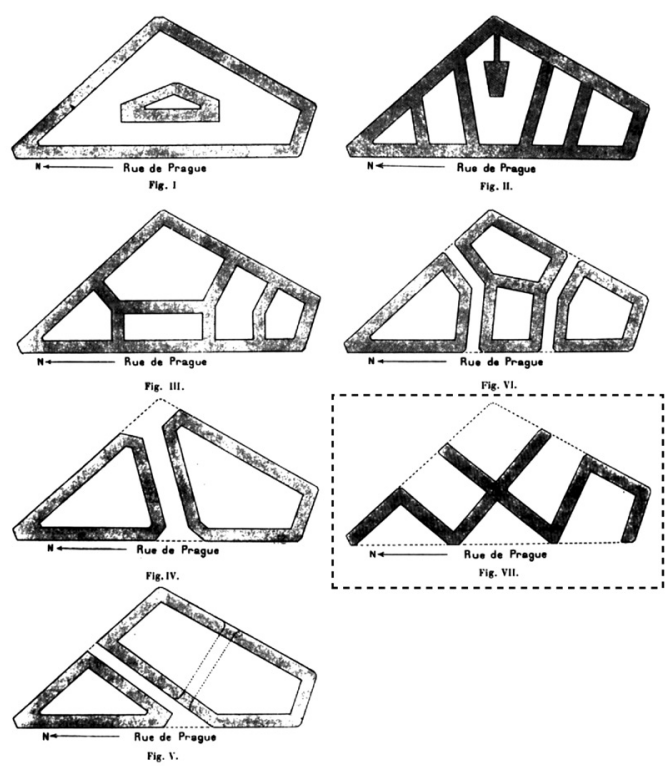

Figura 5. Propuestas de ordenación de una manzana en Rue de Prague, destacando la solución tipológica de Tony Garnier en la esquina inferior derecha. Concurso de la Fundación Rothschild para un edificio plurifamiliar en París, 1905

Fuente: Le Concours Rothschild. (1905)

Esta solución tipológica de Tony Garnier, que enlaza con sus estudios para Une Cité Industrielle (1904-1917), era además convergente con algunas de las propuestas urbanas que Eugène Hénard publicó en su obra Études sur les transformations de Paris entre 1902 y 1909. En concreto con el boulevard à redans, o boulevard retranqueado, y con el que Hénard (1905) adoptó un posicionamiento crítico ante el trazado urbano de Haussman como fuente de insalubridad. Una actitud convergente con la de otros arquitectos coetáneos como Adolphe Augustin Rey o Henri Sauvage y Charles Sarazin, quienes se apoyaron en el contexto favorable de regulación y promoción de la vivienda social en Francia, en activo desde finales del siglo XIX, para ensayar las soluciones higiénicas que había desarrollado la arquitectura sanatorial y dar respuesta al problema de la vivienda obrera a gran escala dentro de la propia ciudad industrial.

\subsection{Hacia un modelo de ciudad saludable en los años veinte}

Aunque las políticas en materia de vivienda social fueran distintas para cada país, todas aspiraban a ofrecer soluciones al problema de la infravivienda, conciliando la salud pública con la vivienda masiva y una adecuada gestión del suelo. La Woningwet o ley de vivienda en Holanda (1901) fue pionera en este sentido, mientras que la Alemania de la posguerra se convirtió en un referente internacional gracias al programa de viviendas de la República de Weimar (1918-1933). Para tomar conciencia de su relevancia solo hay que mencionar los ejemplos de Ernst May para el Departamento de Vivienda de Frankfurt, de Walter Gropius en la Siedlung de Törten (Dessau) y Siedlung Haselhorst (Berlín), o de Bruno Taut y Martin Wagner, entre otros. Suiza o Bélgica tampoco 
se quedan atrás en estas políticas, llevadas a la práctica por arquitectos como Hannes Meyer o Victor Bourgeois, respectivamente.

Evidentemente, todos estos profesionales mostraron interés en aplicar en sus proyectos los avances en materia de salud y prevención de enfermedades, en especial frente a la tuberculosis, así como en materia de estandarización y de racionalización de los procesos constructivos que hacían más asequibles los precios de compra de la vivienda. Para alcanzar este objetivo tomaron como referente a las innovaciones arquitectónicas que avanzaron los sanatorios al inicio del siglo XX (JiménezMorales y Vargas-Díaz, 2017). No obstante, el debut de estos intercambios se remonta a ciertas experiencias realizadas en París, sin duda favorecido por las políticas de vivienda social que se estaban llevando a cabo en Francia desde 1850, entre las que destacaban la Ley Jules Siegfried y la Société Française des Habitations à Bon Marché (HBM), que quedó constituida tras el congreso homónimo de 1889.

En este contexto, los arquitectos Henri Sauvage y Charles Sarazin proponen sus viviendas salubres para la población obrera a partir de una traslación del prototipo de sanatorio escalonado o Freilufthaus que había dado a conocer el doctor Camille Savoire durante su disertación en la École des Beaux Arts de Paris en el año 1902. La confianza que depositaron ambos arquitectos en este sistema, que transformaría tanto el bloque residencia plurifamiliar como la sección transversal de los trazados urbanos post-haussmanianos, les llevó a patentarlo en 1912 (Loyer y Guéné, 1987). En ese mismo año, Sauvage y Sarazin edificaron en la Rue Vavin un primer Groupe d'Habitations à Bon Marché con una fachada escalonada que duplicarían en el bloque piramidal de la Rue des Amiraux (Figura 6). El espacio central que quedaba confinado entre ambos perfiles quebrados aún está ocupado por la piscina y los baños públicos con los que los arquitectos quisieron concluir este bloque-manzana de viviendas higiénicas; un modelo de residencia plurifamiliar con servicios colectivos unificados que fue recurrente en Sauvage a la hora de concebir sus utopías urbanas, tal como sucedió con sus propuestas para el Concours Rosenthal (1931) o Metrópolis (1928), todo un manifiesto futurista para una ciudad contenida dentro de una doble pirámide de escala monumental.

Evidentemente la idea de utilizar la arquitectura escalonada como un recurso formal, ya fuera para la vivienda o la ciudad, no fue original de Sauvage. Borie (1865) o Sant'Elia (1914) ya la habían empleado antes que él en sus obras. El primero, en su utopía urbana Aérodômes para la higienización de la periferia parisina; el segundo, en su Manifesto dell'architettura futurista. Asimismo, el uso de la arquitectura escalonada como opción para limitar la edificabilidad, permitiendo un soleamiento razonable y el aprovechamiento parcelario suficiente, ya aparecía registrada en la Building Zone Resolution de Nueva York en 1916. Para entonces, el arquitecto Tony Garnier persistía en sus bocetos para Une Cité Industrielle (1904-1917) mientras que Adolf Loos ya había concluido la residencia aterrazada para Gustav Scheu (1912). Fue precisamente Loos, como arquitecto municipal de Viena, quien puso de nuevo de manifiesto el papel que podía jugar la arquitectura escalonada para

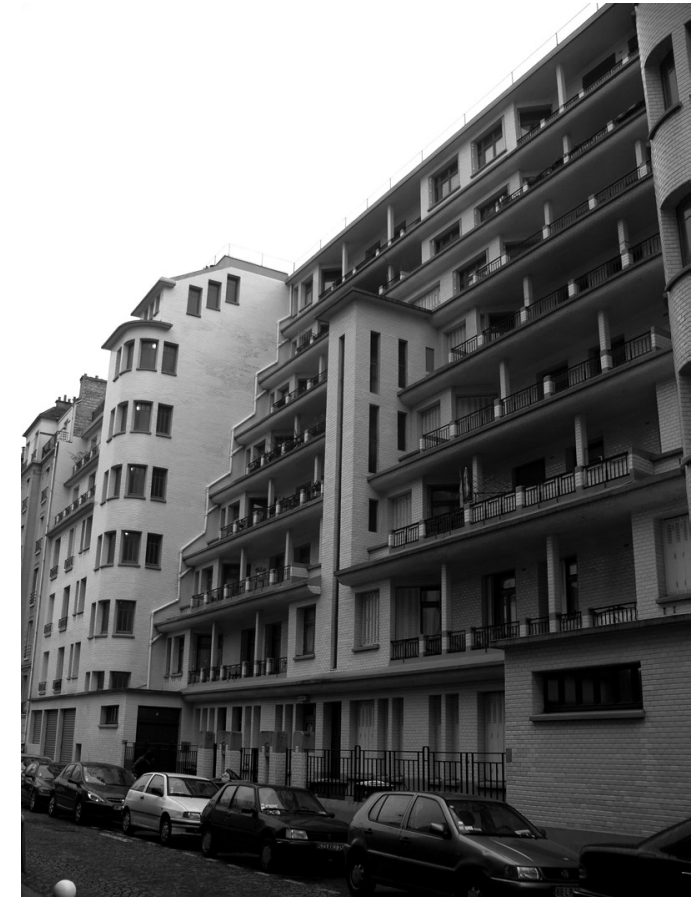

Figura 6: Fachada meridional del inmueble à gradins de Henri Sauvage en la Rue des Amiraux de París, 1923-1925 Fuente: R. Mathis, (2011). Wikimedia Commons. (CC BYSA 3.0)

procurar soluciones habitacionales salubres y, a su vez, compatibles con la alta densidad. Con este objetivo Loos presentó su propuesta de residencia colectiva para familias de escasos recursos en la Inzersdorferstrasse (1923) (Figura 7). La sección escalonada se convertía aquí en calles elevadas que construían una secuencia ascendente de espacios comunes, donde tenía lugar la vida del urbanita sin renunciar al ideal de casa como espacio habitable.

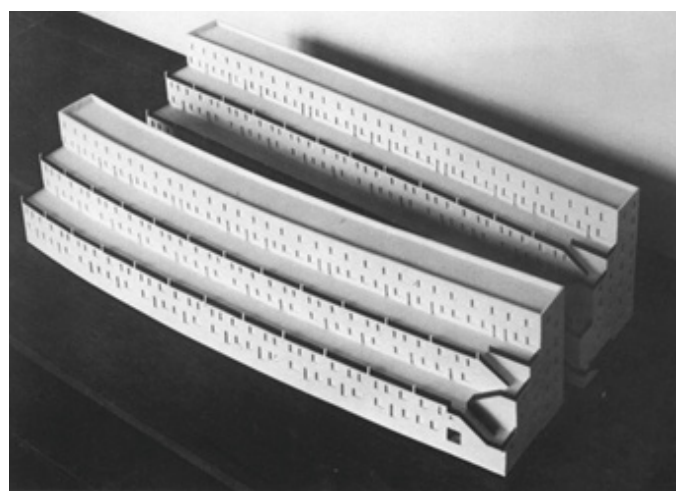

Figura 7: Maqueta de la propuesta de Adolf Loos para una vivienda plurifamiliar en la Inzersdorferstrasse, Viena, 1923

Fuente: M. Nograsek, (2001)

Las posibilidades que ofrecían las arquitecturas de terrazas escalonadas para investigar con la alta densidad poblacional también se tradujo en propuestas que superaban ampliamente la escala de la ciudad tradicional. El Wohnberg Project (1928), por ejemplo, fue concebido por el arquitecto alemán Walter Gropius como una 
mega-estructura utópica en forma de A con 32 niveles de viviendas retranqueadas. Su doble fachada escalonada y su forma piramidal dejaban pocas dudas sobre el influjo de Sauvage o Döcker sobre un diseño que, sin embargo, se situaba en las antípodas de otros modelos urbanos de la época. De hecho, la propuesta de Gropius no salió a la luz hasta 1961, en la exposición Visionary Architecture del MoMA de Nueva York, ayudado por un contexto de ruptura con el Movimiento Moderno y de innovación urbana que despertó un renovado interés por la arquitectura escalonada.

\section{Conclusiones}

En continuidad con lo que había sucedido en el siglo XIX en las primeras décadas del siglo XX ciertos médicos $y$ arquitectos apostaron decididamente por intervenir en los problemas de hacinamiento e insalubridad que sufría la población de la ciudad industrial, densa y contaminada. Todos ellos estaban movilizados en un contexto científico que priorizaba la prevención frente a la cura sanitaria y que promovía un hábitat higiénico, ya fuese individual o colectivo, como fórmula adecuada para hacer frente a determinados males, entre los que destacaba la tuberculosis; una enfermedad que empezaba a ser considerada como un producto socia y que, como tal, su prevención pasaba inexorablemente por un cambio irreversible en las formas arquitectónicas de hacer ciudad. Así lo entendían algunos doctores como Camille Savoire o David Sarason, quienes emplearon la arquitectura hospitalaria más avanzada como referente para promover ese ansiado cambio.

El sanatorio antituberculoso que originalmente fue concebido para la cura de esta grave enfermedad, se instrumentalizó entonces con este fin, procurando así definir un hábitat preventivo con el que transferir las ideas de higiene y salubridad sanitarias al espacio doméstico y urbano. El sanatorio de terrazas escalonadas fue el que inicialmente emplearon médicos y arquitectos para alcanzar este objetivo, especialmente desde que David Sarason presentara en el marco del XIV Congreso Internacional de Higiene y Demografía de 1907 su prototipo de Freilufthaus como paradigma de cómo generalizar el diseño sanatorial a la vivienda colectiva y urbana. Ese momento marcó el comienzo de un prolífico período de experimentación arquitectónica que se intensificó en los años veinte; primero en el ámbito de la residencia y, a continuación, a escala de ciudad. Así lo atestiguan las propuestas de Richard Döcker, Adolf Loos, Henri Sauvage, Tony Garnier o Walter Gropius.

No cabe duda que el sanatorio, como institución, está hoy por hoy obsoleto, sobre todo si consideramos que sus terrazas escalonadas fueron la respuesta arquitectónica a unas prácticas médicas que ahora están igualmente extintas. Sin embargo, también hay que reconocer que su impacto en el diseño de la vivienda colectiva contemporánea aún persiste, una vez que recuperó buena parte del crédito perdido tras el final de la Segunda Guerra Mundial. Desde entonces, las posibilidades que ofrecía la tipología arquitectónica escalonada para hacer frente a la escala urbana han sido muy cuestionadas, aunque aún mantiene su posición aventajada en el campo de la investigación en materias de vivienda y urbanismo; una cualidad que posiblemente salga reforzada un siglo después, en medio de una nueva pandemia global, cuando las medidas profilácticas implementadas por las autoridades sanitarias para la contención del Covid-19 están desafiando la esencia misma de las ciudades.

\section{Agradecimientos}

Esta investigación se ha realizado en el marco del Programa UMA FC 2015-004 para el Fortalecimiento de RR. HH. en Investigación de la Universidad de Málaga, financiado por la Universidad de Málaga y Andalucía TECH.

Cómo citar este artículo/How to cite this article: Jiménez-Morales, E. y Vargas-Díaz, I. (2021). Buscando arquitecturas para una ciudad saludable. El caso del sanatorio escalonado en los años veinte. Estoa. Revista de la Facultad de Arquitectura y Urbanismo de la Universidad de Cuenca, 10(20), 7-16. doi: https://doi.org/10.18537/est.v010.n020.a01 


\section{Referencias bibliográficas}

Bernhard, O. (1917). Sonnenlicht Behandlung in der Chirurgie. Deutsche Chirurgie Series. (Vol. 23). Ferdinand Enke.

Borie, H. J. (1865). Aérodômes: essai sur un nouveau mode de maisons d'habitation. Applicable aux Quartiers les plus mouvementés des grandes Villes. Morris et Compagnie.

Campbell, M. (2005). What Tuberculosis did for Modernism: The Influence of a Curative Environment on Modernist Design and Architecture. Medica History, 49(4), 463-488. https://doi.org/10.1017/ S0025727300009169

Colomina, B. (2019). X-ray Architecture. Lars Muller Publishers

Cremnitzer, J. B. (2005). Architecture et santé, le temps du sanatorium en France et en Europe. Picard.

Cremnitzer, J. B., y Toulier, B. (Eds.) (2008). Histoire et Réhabilitation des Sanatoriums en Europe. Docomomo.

Döcker, R. (1929). Terrassentyp: Krankenhaus, Erholungsheim, Hotel, Bürohaus, Einfamilienhaus, Sieglungshaus, Miethaus, und die Stadt. F. Wedekind \& Co.

Duarte, I. (2005). Sanatorios para tuberculosis: auge y decadencia. Ars. Médica, Revista de Estudios Médicos Humanísticos, 2(34), 170-185. https://doi.org/10.11565/ arsmed.v34i2.216

Dumont, M. J. (1991). Le logement social à Paris 1850 1930: les habitations à bon marché. Luik Mardaga.

Faust, B. C. (1829). Andeutungen über das Bauen der Häuser und Städte zur Sonne. Hahn'sche Hofbuchhandlung.

Fondation Miremont. (2017). Dr. Rollier-Heliotherapie Leysin. 1911. [Fotografía]. Wikimedia Commons. https://commons.wikimedia.org/wiki/File:Dr_rollier_-_ heliotherapie_-_leysin.jpg

Garnier, T. (1917). Une cité industrielle. Étude pour la construction des villes. Auguste Vincent.

Gescheit, H. (1929). Neuzeitliche Hotels und Krankenhäuser; ausgeführte Bauten und Entwürfe. Ernst Pollak Verlag.

Giedion, S. (1929). Befreites Wohnen. Licht, Luft, Öffnung. Orell Füssli.

Godin, J. B. (1871). Solutions Sociales. Le Chevalier Editeur.

Grandvoinnet, P. (2020). Sanatoriums in Europe: Build Heritage and Transformation Strategies. Docomomo Journal, Cure and Care, (62), 44-51. https://dx.doi. org/10.52200/62.A.IYJYY4X1
Grober, J. (1932). Das deutsche Krankenhaus: Handbuch für Bau, Einrichtung und Betrieb der Krankenanstalten. Gustav Fischer.

Hénard, E. (1905). Études sur les transformations de Paris. Librairies-imprimeries réunies.

Howard, E. (1898). To-morrow: A Peaceful Path to Real Reform. Swan Sonnenschein \& Co.

Iglesias Picazo, P. (2011). La habitación del enfermo. Ciencia y arquitectura en los hospitales del Movimiento Moderno. Fundación Caja de Arquitectos de Barcelona.

Jiménez-Morales, E. (2014). El hotel turístico. Viaje a los orígenes de su arquitectura, 1693-1932 (Tesis doctoral, Universidad de Málaga) https://riuma.uma.es/xmlui/ handle/10630/8067

Jiménez-Morales, E., y Vargas-Díaz, I. C. (2017) Hotels and sanatoria: the influence of tuberculosis on mass tourism architecture. Historia ciencia saudeManguinhos, 24(1), 243-260. https://doi.org/10.1590/ s0104-59702017000100006

Knauff, F. (1879). Das neue akademische Krankenhaus in Heidelberg. Bassermann

Le Concours Rothschild. (1905). La construction moderne, 49, 338-339.

Loyer, F., y Guéné, H. (1987). Henri Sauvage. Les inmeubles a gradins. Mardaga.

Lüthi D. (2005). The influence of Good air on architecture. A « Formal Cure»? The appearance of the Alpine sanatorium in Switzerland, 1880-1914. Revue de géographie alpine, 1(93), 53-60. https://www.persee.fr doc/rga_0035-1121_2005_num_93_1_2332

Mathis, R. (2011). Immeuble et piscine des Amiraux (Architecte : Henri Sauvage) [Fotografía] Wikimedia Commons. https://upload.wikimedia.org/wikipedia/ commons/b/b5/Immeuble_Sauvage___piscine_des amiraux__rue_des_amiraux.JPG

Medici T. C. (2003). Die tuberkulose und das moderne wohnideal. Praxis, 34(92), 1382-1391. https://doi. org/10.1024/0369-8394.92.34.1382

Megahed, N. A., y Ghoneim, E. M. (2020). Antivirus-built environment: Lessons learned from covid-19 pandemic. Sustainable Cities and Society, 6, 102350. https://doi. org/10.1016/j.scs.2020.102350

Miller, Q. (1992). Le sanatorium: architecture d'un isolement sublime. Ecole Polytechnique Federale de Lausanne Department D’Architecture.

Nograsek, M. (2001). Modell eines ArbeiterTerrassenhauses mit 2 geschoßigen Kleinwohnungen an Hochstrassen für Wien 1923. [Fotografía] Wohnwert Werturteile im Vergleich an ausgewählten Wohnanlagen in Graz, (Tesis doctoral, Technischen Universität Graz) https://docplayer.org/31012655-Wohnwert-werturteileim-vergleich-an-ausgewaehlten-wohnanlagen-in-grazband-1.htm 
Overy, P. (2008). Light, Air and Openness: Modern Architecture Between the Wars. Thames \& Hudson.

Poeschel, E. (1928). Das Flache Dach im Davos. Das Werk (15), 102-109

Rey, A. A., Pidoux, J., y Barde, C. (1928). La Science des Plans de Villes. Dunod.

Rollier, A. (1923). Heliotherapy: with special consideration of surgical tuberculosis. Frowde and Hodder \& Stoughton.

Sant'elia, A. (1914). ॥ Manifesto dell'architettura futurista. Rivista Lacerba, 1(15), 167.

Sarason, D. (23-29 septiembre de 1907). Ein neues Bausystem für Krankenanstalten und Wohnhäuser Bericht über den XIV. internationalen Kongress für Hygiene und Demographie, Berlín, Alemania.

Sarason, D. (1909). Freilufthäuser. Ein neues Bausystem für Krankenanstalten und Wohngebäude (Prämiert mit der goldenen Medaille der Intern. Tuberkulosekongress in Washington, 1908. Zeitschrift für Krankenanstalten, (12), 280-290

Sarason, D. (1913). Das Freilufthaus, Ein Neues Bausystem Für Krankenanstalten Und Wohngebäude: Prämiiert Mit Der Goldenen Medaille Vom Internationalen Tuberkulosekongress In Washington 1908. J. F. Lehmann. https://archive.org/details/bub_gb_OgoTAQAAMAAJ/ page/n13/mode/2up

Savoire, C. (1902). La lutte antituberculeuse, ses rapports avec l'art de l'architecte, de l'ingénieur et de l'industrie technique. Bulletin de la Société des architectes diplômés par le gouvernement, 3(4), 133-134.

Sonne, W. (2009). Dwelling in the metropolis: Reformed urban blocks $1890-1940$ as a model for the sustainable compact city. Progress in Planning, 72(2), 53-149.

Vogt, A. (1879). Über die Richtung der städtischen Straßen nach der Himmelsgegend und das Verhältnis ihrer Breite zur Häuserhöhe. Zeitschrift für Biologie. 